Ophthalmologe 2010 · 107:997-998

DOI 10.1007/s00347-010-2157-0

Online publiziert: 29. September 2010

(c) Springer-Verlag 2010

\author{
S. Schmitz-Valckenberg · F.G. Holz \\ Universitäts-Augenklinik Bonn
}

\title{
Geographische Atrophie bei AMD
}

Berer Netzhauschichten, auch eine der möglichen Ursachen für Sehverlust in Gegenwart einer feuchten AMD unter Anti-VEGF-Therapie.

Insofern besteht ein erheblicher medizinischer Bedarf für eine wirksame Therapie. Dabei bietet die Entwicklung einer erfolgreichen Behandlung der geographischen Atrophie auch die Aussicht auf einen früheren Einsatz entsprechender Medikamente zur Vermeidung oder zumindest zum Hinauszögern von sehmindernden Spätformen der AMD überhaupt. Es geht also um nicht weniger als den „heiligen Gral“ der AMD-Therapie.

Erfreulicherweise konnte das Verständnis über relevante ,pathways" bei der multifaktoriellen Erkrankung dank interdisziplinärer Bemühungen in den letzten Jahren erheblich erweitert werden. Mit Übersichten zu verschiedenen Aspekten wollen wir einen aktuellen Überblick geben.

Im Beitrag von Brinkmann et al. werden Aspekte klinischer Merkmale der geographischen Atrophie beleuchtet. Insbesondere liefern Daten von longitudinalen Beobachtungen wichtige Erkenntnisse zum natürlichen Verlauf und relevanten prädiktiven Faktoren der Erkrankung. Aufschluss leistete hier ein auch im Rahmen des DFG-Schwerpunktprogramms AMD gefördertes Verbundprojekt in Deutschland mit der multizentrischen FAM-Studie (FAM: Fundusautofluoreszenz bei altersabhängiger Makuladegeneration), bei der Patienten über viele Jahre mit dieser insgesamt relativ langsam fortschreitenden Erkrankung systematisch untersucht wurden $[2,3]$. Hier zeigte sich auch, dass die Progressionsraten zwischen den Patienten sehr unterschiedlich sein können und sich - basierend auf der
cSLO-gestützten Bildgebung (cSLO: konfokale Scanning Laser Ophthalmoskopie) Biomarker erfassen lassen, die prädiktive Hinweise auf die Ausdehnungsgeschwindigkeit der Atrophie geben. Im Beitrag von Fleckenstein et al. wird der zentrale Stellenwert der FundusautofluoreszenzBildgebung, aber neuerdings auch der spektralen optischen Kohärenztomographie (,spectral domain“) bei der geographischen Atrophie aufgezeigt.

\section{- Die zentrale Herausforderung bleibt die Entwicklung effektiver und sicherer Behandlungsansätze.}

Im letzten Beitrag werden Paradigmen und potenzielle "targets", basierend auf Einblicke in relevante molekulare Mechanismen, dargestellt. Dabei wäre das zunächst bescheidene Therapieziel, dessen Realsierung allerdings bereits einen bedeutenden Durchbruch darstellte, die Progression der Erkrankung zu verlangsamen, analog zur gegenwärtigen Glaukomtherapie. Ein Novum dieser klinischen Studien stellt der auch mittlerweile erstmals von der FDA (Food and Drug Administration) in den Vereinigten Staaten von Amerika akzeptierte Hauptauswertungsparameter dar: Im Gegensatz zum funktionellen Endpunkt (Sehschärfe) wird der Therapieeffekt primär an einem anatomischen Endpunkt gemessen, d. h. der Veränderung der Fläche der betroffenen atrophischen Areale. Dies wird notwendig, da der zentrale Visus nicht unbedingt aussagekräftig ist, wenn beispielsweise die Foveola von dem Krankheitsprozess zunächst ausgespart bleibt, was ein typisches Merkmal bestimmter Verlaufsformen der geographischen Atrophie ist. Viele Ansätze befinden sich in vorklinischen und 
bereits in klinischen Entwicklungsphasen. Es besteht also Grund zum Optimismus, dass den betroffenen Patienten schon bald eine wirksame Therapie angeboten werden kann, die über die bis dahin rein rehabilitativen Maßnahmen hinausgeht.

Ihre

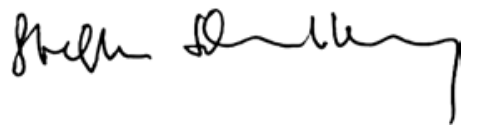

Steffen Schmitz-Valckenberg

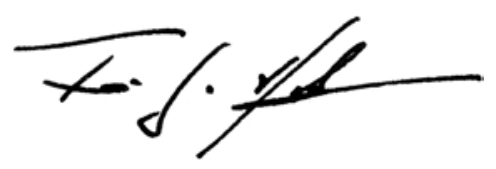

Frank G. Holz

\section{Korrespondenzadresse}

\section{PD Dr. S. Schmitz-Valckenberg}

Universitäts-Augenklinik Bonn

Ernst-Abbe-Straße 2, 53127 Bonn

steffen.schmitz-valckenberg@ukb.uni-bonn.de

\section{Literatur}

1. Augood CA, Vingerling JR, de Jong PT et al (2006) Prevalence of age-related maculopathy in older Europeans: the European Eye Study (EUREYE). Arch Ophthalmol 124:529-535

2. Fleckenstein M, Adrion C, Schmitz-Valckenberg $S$ et al (2009) Concordance of disease progression in bilateral geographic atrophy due to AMD. Invest Ophthalmol Vis Sci 51:637-642

3. Holz FG, Bindewald-Wittich A, Fleckenstein M et a. (2007) Progression of geographic atrophy and impact of fundus autofluorescence patterns in agerelated macular degeneration. Am J Ophthalmol 143:463-472

4. Klein R, Klein BE, Knudtson MD et al. (2007) Fifteen-year cumulative incidence of age-related macular degeneration: the Beaver Dam Eye Study. Ophthalmology 114:253-262

\section{Tabandeh H, Goldberg MF} The Retina in Systemic Disease A Color Manual of Ophthalmoscopy Thieme Verlag 2010, 366 S., 350 Abb., (ISBN 978-1-60406-055-3), 51.00 EUR

Das in englischer Sprache geschriebene Taschenbuch „The Retina in Systemic Disease. A Color Manual of Ophthalmoscopy" von H. Tabandeh und M.F. Goldberg umfasst 366 Seiten und ist mit über 300 hochwertigen farbigen Fundusaufnahmen illustriert. Für den ungeübten Ophthalmologen mit wenig Erfahrung in der Fundusuntersuchung ist es ein praktisches Handbuch, um die Ophthalmoskopie zu erlernen und einen Überblick über retinale Manifestationen bei systemischen Erkrankungen zu gewinnen.

Es ist in drei Abschnitte unterteilt. Im ersten Teil wird zunächst die Anatomie und Physiologie der Netzhaut in groben Zügen rekapituliert. Daran anschließend werden dem ungeübten Untersucher Schritt für Schritt die Grundlagen und Prinzipien der direkten und indirekten Funduskopie mithilfe von klaren Illustrationen erklärt.

Zahlreiche praktische Hinweise unterstützen den Leser dabei, die Visualisierung zu optimieren.

Der zweite Abschnitt bietet einen allgemeinen Überblick über verschiedene retinale Manifestationen, welche durch gutes Bildmaterial reichhaltig illustriert werden. Zu den einzelnen retinalen Zeichen werden stichpunktartig mögliche assoziierte systemische Erkrankungen aufgelistet. Im dritten Abschnitt werden aus dem weiten Feld der systemischen Erkrankungen einzelne Gruppen herausgegriffen und die wichtigsten retinalen und ophthalmologischen Manifestationen präsentiert. Jede Erkrankung ist mit einer kurzen Beschreibung, den typischen Fundusveränderungen und möglichen Differenzialdiagnosen versehen, welche den Kliniker schnell mit nützlichen ophthalmologischen Grundinformationen versorgen.

Auf eine weiterführende Diagnostik mit möglichen ophthalmologischen Spezialuntersuchungen mit OCT, Elektrophysiologie, Angiographie oder Autofluoreszenz, wie auch auf mögliche Therapie- und Behandlungsoptionen, wird nicht einge- gangen. Besonders Ärzte im Bereich der Neurologie, Rheumatologie, Pädiatrie, Inneren Medizin und Allgemeinmedizin werden Freude an dem Taschenbuch mit zahlreichen relevanten Fundusbeispielen finden.

C.H. Meyer (Bonn) 